\title{
A Mining Frame Work of CO-PO Attainment using Deep Learning Techniques
}

\author{
P Vasanth Sena, P Sammulal, Suresh Pabboju, D L Srinivasa Reddy
}

\begin{abstract}
CO-PO (Course Outcome and Program Outcome) attainment for OMR based answer sheet automation playing very curtail role in pupil concert analysis in this approach. In the proposed work, marks evaluation sheet is consider as input image, then apply frame cropping technique to extract the marks filled table by subdividing into cells as individual images by frame cropping technique. In order to recognition of hand written digit in each frame, various machine learning models are adopted, trained. Experimental results from proposed work show that convolutional neural network excels higher in identification digits from frames. The outputs are then converted to CSV version, which is used to evaluate CO-PO attainment for each learner. The experiments have been conducted and tested in proposed work on various machine learning techniques and compared the results to pick the optimal model.
\end{abstract}

Keywords: Hand written digit, Object detection, Classification, Deep learning.

\section{INTRODUCTION}

Intake of programs in education institutions becoming increasing every year. Evaluations of student performance in the form of outcomes, which are mapped with course outcomes, become more important than ever before. The requirement for mining frame work for CO-PO attainment and fraud detection in scrutiny and marks counting reduce the risk factor and increases efficiency. Course outcomes are available in syllabus for any course. Based on CO-PO attainment Program Outcomes are assessed. Program Outcomes are designed from Graduate outcomes. Graduate outcomes are twelve which are defined by national board of accreditation (NBA). These twelve graduate outcomes summarized to program outcomes for any program.

Convolutional Neural Networks are popular for image processing. $\mathrm{CNN}$ can also be used for translating these images to text and numbers. CNN is the class of deep learning. Framework is a graphical representation of a set of classes and interfaces using Application programing Interface (API). Proposed work one among several offerings of image detection and diagnosis techniques look into

innovative methods like Frame Cropping, CNN, RNN, LSTM model

Revised Manuscript Received on March 18, 2020.

* Correspondence Author

P.Vasanth Sena*, Asst Professor, IT department, CBIT, Hyderabad, INDIA. Email: vasanthasena_it@cbit.ac.in

Professor Sammulal Porika, CSE department, JNTU CEJ, Hyderabad, India. Email: sammulalporika@gmail.com

Professor Suresh Pabboju, IT department, CBIT, Hyderabad, India Email: plpsuresh@gmail.com

Dr D L Srinivasa Reddy, IT department, CBIT, Hyderabad, India. Email: dlsrinivasareddy_it@cbit.ac.in etc.. and judging the right one, that could give the best results.

In proposed work, the deep learning classification models are trained and tested on the MNIST hand written digit dataset, which is freely available.

This work has been divided into six sections. Section II briefly explains the existing work. Section III describes the proposed. Section IV deals with the prepossessing stage and the different implementation methods. Section V discuses about results and comparisons about performance levels in different aspects, Conclusion and future enhancement are briefed in the last section.

\section{RELATED WORK}

Direct Assessment Measures [3] is used to estimate the student performance by the LSTM algorithm. Its precision is 85.5 percent only. Author predicts the Achievements, outcomes and proposal for global accreditation of engineering education [1] using binary classifier C4.5 algorithm. The accuracy of this technique is 82.5.

This case study [2] was based on support vector regression technique, in this approach so may anomalies were detected while preprocessing the dataset. The research article entitled with Achievement of Program Outcomes Using Assessment Plan Measurement student performance Analysis for Detecting and locating weak students in particular course outcome using ANN and SVM [4] are used support vector machine and artificial neural network techniques. Belsito et al. [6] developed artificial neural network method for slow learner student identification.

\section{METHODOLY}

\section{A. Architecture of Convolution Neural Network}

1) Bone of CNNs: The Fig 3.1 exhibits a widespread architecture of the CNN model, consisting of two convolutional operations, two operations of pooling that gives rise to four groups of feature maps, and the fully connected layer in the end. The last layer, being a full connection layer, obtains the input data by flattening all elements of the fourth group of feature maps. Full connection layers are constantly stack with each other in the tail and remaining convolutional and pooling layer can be mixed to stack together at the head of the architecture. The statistics in Fig. 3.1 refer to the dimensions of the equivalent layer. Mainly, the input data is with $24 \times 24$ pixels, the output is with $128 \times 1$ vector, and the other numbers represent the feature map outlines. For example, $4 @ 20 \times 20$ indicates there are 4 feature maps, each with the size of $4 \times 4$.

Published By:

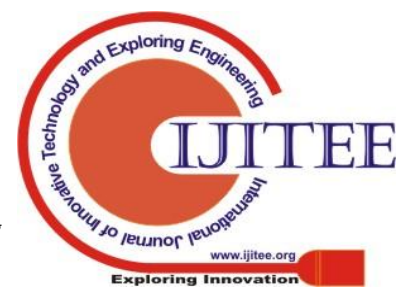




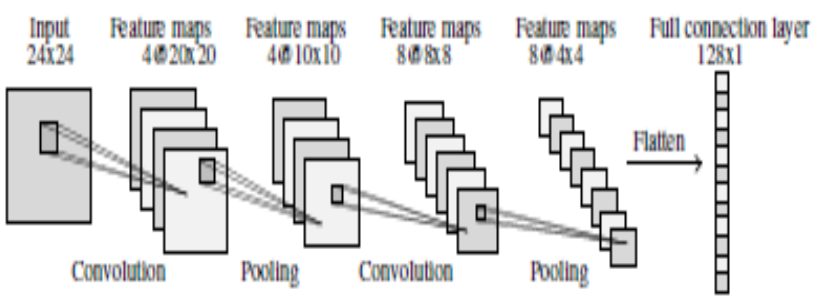

Fig3.1: Architecture of Convolution Neural Networks

Adding a bias term the system can get convolutional results regarding each filter and then through nonlinearity, such as the Rectifier Linear Unit (ReLU), before they are warehoused into the feature map. Clearly, the involved parameters in one convolutional operation are the filter height and filter width, the number of feature maps, the stride height and stride width, the type of convolutional and the connection weight in the filter. The pooling operation except for the element-wised product be similar to the convolution operation and the resulted values of the corresponding feature map. Concisely, the pooling operation services a predefined window, it is the kernel which is used to gather the average value or the highest value of the elements where it slides, and the slide size is also called "stride" as in the convolutional operation.

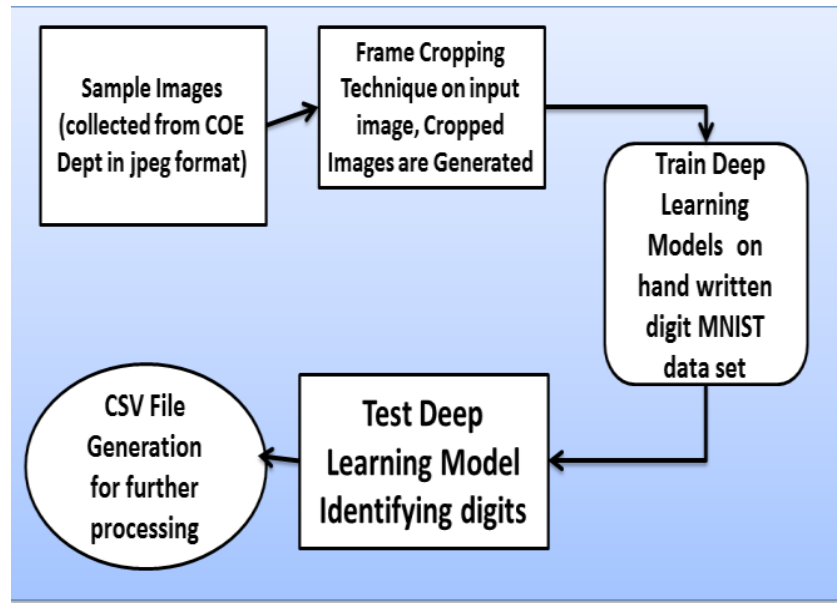

Fig 3.2: Block diagram of the proposed Model

The methodology part alienated into various categories like existing methods, proposed approach and challenges faced on collecting of data sets.

In existing method optical mark recognition (OMR) is the technology of electronically extracting intended data from marked fields, such as checkboxes and fill-infields, on printed forms. OMR technology scans a printed form and reads predefined positions and records where marks are made on the form, but it unable to recognize the hand written digits.

In proposed approach data is read from OMR JPG image and applying frame cropping technique and Deep learning model to identify handwritten digits.

The major Challenges faced On Collecting hand written Dataset for testing purpose is very challenging issue. writing politely letters to concerned authorities through proper channel. It is a time taking process in our case, even it last 4 to 5 years data, So many security ans authentication issues are softly handled, for training MNIST data set is adopted.

\section{IMPLEMENTATION}

In the implementation phase the following steps plays a very important role. They are as follows.

\section{A. Algorithm to Identify Hand Written Digits:}

1) Collect paper valuation samples from COE. The samples are .jpg format.

2) Extract part-A, part-B and part-II sub parts like total marks and serial no of answer book in the bundle by applying image crop techniques. Java implementation is done for this part.

3) In part-II division, Applying image processing techniques get marks and SNO of answer booklet in bundle with filled circles.

4) In Part-A and part-B applying image crop techniques, Sub divided into each and every cell.

5) Hand written digit recognition (MNIST dataset/ EMNIST) CNN trained model is used to identify marks in above cell.

6) Generate CSV file for above marks.

7) Applying prototype of marks counting, check whether the marks are identical in both parts. (part-A + Part-B marks generated by model and marks generated by bubbling circles in total marks area)

8) If both marks are same, it indicates scrutiny did his marks counting duty perfectly; otherwise mismatch is marks counting and provision to fraud in scrutiny marks counting.

9) Generated CSV File is also used for CO-PO attainment for marks.

10) In part- $B$ area, the cell contains marks for its sub parts like $4+5+3$. It is very interesting issue, it is addressed with the help of image cropping techniques.

11) One more crucial functionality is Extract the exact cell part.

\section{B. Procedure to construct CNN model :}

Convolutional neural network model is the neural network approach; it is configured with the following method. First and foremost step is the importing required sequential libraries to our model. The MNIST data also added from keras datasets library. The built in MNIST handwritten digits data set has a training set of 60,000 examples, and a test set of 10,000 examples is used in our experiment.

The next step is Load the mnist data set into our model as labelled X_train, y_train, X_test, y_test. Reshape our image into $28 \mathrm{X} 28$ pixels as floating point data, the parameters are sample images, width, heights, channel whether it is colored, gray scale or black and white. Normalize the input samples from 0-255 pixel values to in the range of 0,1 both inclusive. One hot encode outputs is used for the class labeled as y_train, y_test and reshaping. The further extending is creating a simple sequential CNN model with the following configuration by adding the following methods in sequential order as follows.

Conv2D function is added to our model using the parameters as input shape $28 \mathrm{X} 28$ black and white image with relu activation function, then MaxPooling2D() function is added to our model. The functions are repeated twice for getting two layers.

Then $20 \%$ percentage of results are dropped out by by invoking Dropout(0.2) function. Inorder to smoothen the results the Flattern() function is invoked. The function Dense(128, activation='relu') is used for further relu activation function.

\section{Published By:}


Then $50 \%$ percentage of results are dropped out by by invoking Dropout $(0.5)$ function. At the end of the program the function Dense(num_classes, activation='softmax') is invoked with softmax as activation function.

The Compilation model is takes place with adam optimizer to reduce the loss of categorical_crossentropy and build the model. In order to fit the model the following procedure is used with epochs 10 and batch_size $=256$.

model.fit $\left(X \_\right.$train, y_train, validation_data=(X_test, y_test $)$, epochs $=10$, batch_size $=256$ )

Finally evaluation of the model takes place and model is saved as model.h5 files and the accuracy of the model is 97.8 percentage.

In predict module the $\mathrm{h} 5$ model is used to predict the given image as hand written digit. Here one interesting thing is that the MNIST hand written image data set is black back ground and white digit but one image is reverse manner i.e white background and black text, So the system uses the following function for required conversion.

ret,img=cv2.threshold(img,127,255,cv2.THRESH_BINARY_INV) The gray scale image open as black and white by $i m g=c v 2 . i m r e a d(' t e s t 1 . j p g$ ',0) and resize into 28X28 matrix.

Table 4.1 various algorithms and its Precision

\begin{tabular}{|l|l|}
\hline Algorithm & Accuracy \\
\hline SVM & 82 \\
\hline Multinomial Naive based (MNB) & 67 \\
\hline Logistic Regression (LR) & 83.5 \\
\hline Random Forest Ensemble method (RFEM) & 86.5 \\
\hline CNN & 97.8 \\
\hline
\end{tabular}

\section{RESULTS AND ANALYSIS}

These are the most significant statistical find outs in recognition of digits. They include:

1) The average section performance is predicted as 68.78 percentages for semester is evaluated from the generated data sets.

2) The failure percentage decreased from first year on average (69.5) to final year (96.8) evaluated from the generated data set.

3) Programing skills learning increase from first years to final year students based on the computing marks percentage in the labs from beginning semesters to final semesters.

4) The bloom's taxonomy different level could be evaluated and used to identify slow learners, medium learners and fast learners and their interesting areas are also easily predicted to their carriers growth levels.

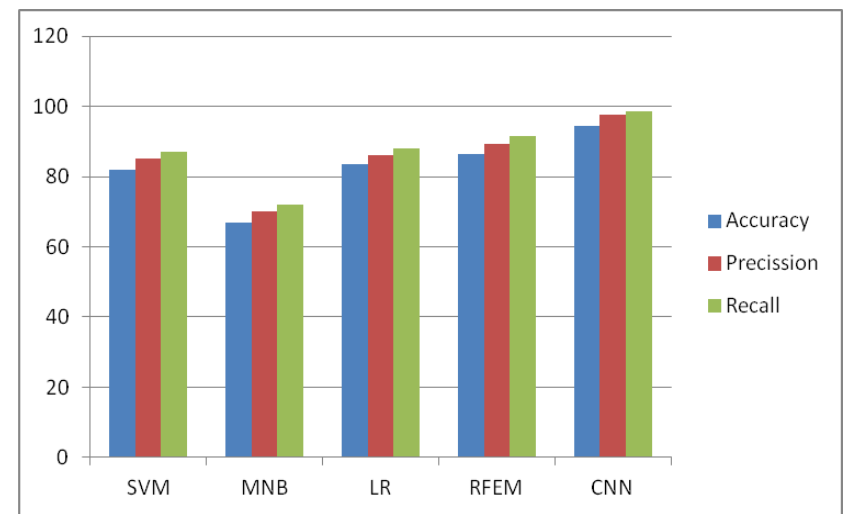

Fig 4.1: Bar Chart representation for classification methods

In order to apply the multinomial naive based technique on data set of size 98000 thousand samples. It predicts the 67 percentage classified accuracy.

A Support Vector Machine (SVM) is a discriminative classifier that the distance between the supporting vectors and the hyper plane are as far as possible i.e optimal hyper Plane. Logistic regression is a classification algorithmic technique used to assign observations to a discrete set of classes. Logistic regression transforms its output using the logistic sigmoid function to return a probability value. In our mission, considering of the logistic regression technique on data set of size 98000 thousand samples. It calculate the 83.5 percentage classified accuracy.

Random forest classifier built a set of decision trees from randomly selected subset of training set. aggregate of many decision trees reduce the effect of noise giving more accurate results.

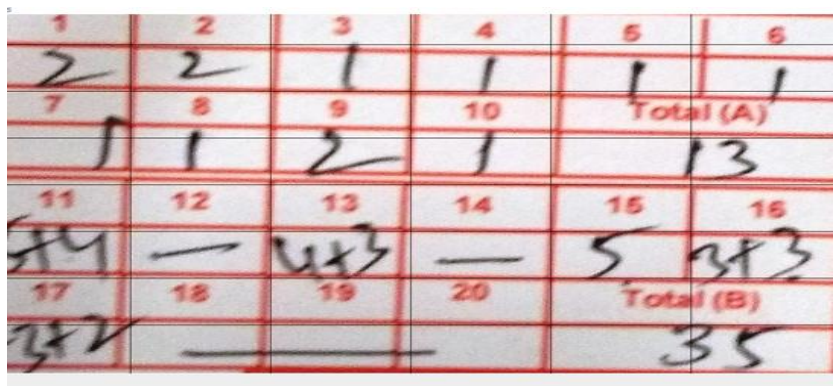

Fig 4.2: Frame Cropping Sample output Screen

In Fig 4.2 the omr marks sheet is consider as the input of the frame cropping methods, it is divides 8 rows and 6 columns.

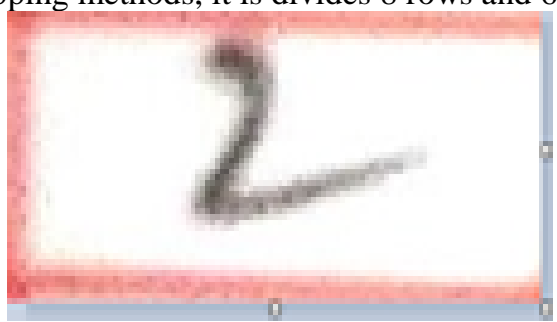

Fig 4.3: Input Image of Convolutional neural network Model

In Fig 4.3 the image supplies as the input our model then the image preprocessing takes place as the first step, and it is feed into input of our model then the model correctly predicted is as the digit 2 . 


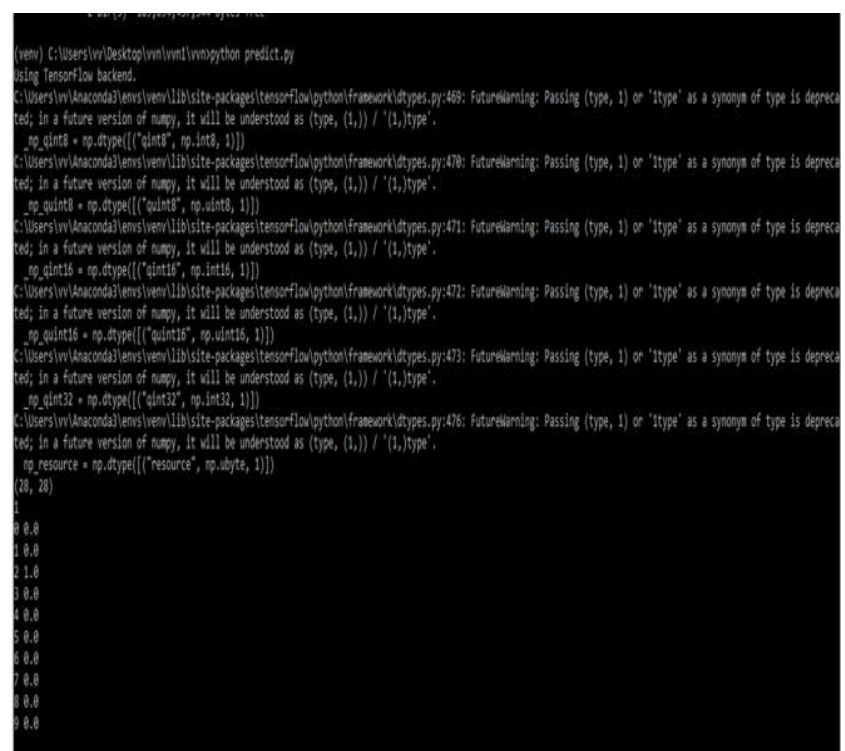

Fig 4.4: Predicted output of CNN Model

In Fig 4.4 the CNN model correctly predicting the input image as 2 with probability 100 percentage and remaining digits as 0 percentages probabilities.

\section{CONCLUSION AND FUTURE ENHANCEMENT}

The MNIST data samples are gathered from MNIST website. The system has trained by various machine learning and deep leaning techniques and model has generated. It has used to predict to the newly incoming hand written samples are digit 0 to 9 . This model can easily predict hand written digits. It can check any counting mistakes. Analyze the student learning and grasping levels.

One more curtail functionality enhanced in future is extract the exact cell part is still need to be optimize.

\section{REFERENCES}

1. R A Abdullah, O K Rahmat, "Achievement of Program Outcomes Using Assessment Plan”, Procedia- Social and Behavioral Sciences, 18 (2011), 87-93.

2. A A Mutalib, R A A Rahmat, A K A Rashid, F Suja, Suraya Sahril, "Measurement and Evaluation of Program Outcomes in the Civil

3. Characterization of an acoustic wireless sensor for water leakage detection in underground pipes By Abdullah Kadri.

4. Anshul Bansal, Susheel Kaushik Rompikuntla, Jaganadh Gopinadhan, Amanpreet Kaur, Zahoor Ahamed Kazi "Energy Consumption Forecasting for Smart Meters".

5. Belsito, S., Lombardi, P., Andreussi, P., \& Banerjee, S. (1998). Leak detection in liquefied gas pipelines by artificial neural networks. AICHE Journal, 44(12).

6. Mpesha, W., Gassman, S. L., \& Chaudhry, M. H. (2001). Leak detection in pipes by frequency response method. Journal of Hydraulic Engineering, 127, 134-147.

7. Misiunas, D. (2005). Failure monitoring and asset condition assessment in water supply system. $\mathrm{PhD}$ Thesis. Department of Electrical Engineering and Automation, Lund University, Sweden.

8. Feng, J., Zhang, H., \& Liu, D. (2004). Application of Fuzzy Decision-Making in Pipeline Leak Localization. Budapest, Hungary: IEEE. 25-29.

9. K. Boggiano and T. S. Pittman, Achievement and motivation: A social-developemental perspective. Cambridge University Press, 1992.

10. Shaeiwitz, Joseph, and Daina Briedis, "Direct Assessment Measures", Paper read at American Society of Engineering Education, 24 - 27 June, Honolulu, Hawaii, 2007.

11. M. Granito and E. Chernobilsky, "The Effect of Technology on a Student' s Motivation and Knowledge Retention," NERA Conf. Proc. 2012, no. 17, 2012.

12. P. Saettler, The Evolution of American Educational Technology. Englewood, Co: Libraries Unlimited, 1990.
13. P. Dillenbourg and J. Sanna, "The Evolution of Research on Computer-Supported Collaborative Learning," Technol. Learn., pp. 3-19, 2009

14. S.-K. Wang and T. C. Reeves, "The Effects of a Web-Based Learning Environment on Student Motivation in a High School Earth Science Course,” Educ. Technol. Res. Dev., vol. 54, no. 6, pp. 597-621, Nov. 2006.

\section{AUTHORS PROFILE}

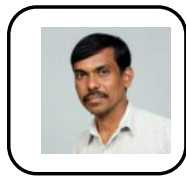

Mr. P. Vasanth Sena, pursuing Ph.D. from JNTU Hyderabad under the faculty of Computer Science and Engineering with data Mining specialization, Done his Post-Graduation from the School of Information Technology, Graduation degree under JNTU and Qualified in APSET July-2012. He is working as Assistant Professor in Chaitanya Bharathi Institute of Technology, under the Department of Information Technology, Previously worked in various Engineering colleges; He had 12 years of Teaching Experience. $\mathrm{He}$ published 10 papers in reputed international journals and 2 in national journals.

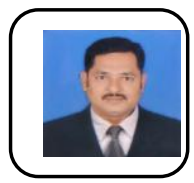

Dr Sammulal Porika, working as professor in CSE dept in JNTU college of Engineering Jagitial. His research interest includes data Mining, Parallel Processing and recommender systems. He published several papers in reputed national and international journals.

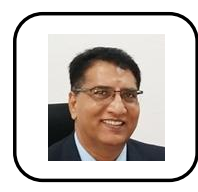

Dr. Suresh Pabboju, born on August 15, 1965 graduated from CBIT, Osmania University and Post Graduated from JNT University, Hyderabad, India. He was awarded $\mathrm{Ph} . \mathrm{D}$. degree in CSE for his research work in the area of Image Processing and Computer Vision by the Osmania University. $\mathrm{He}$ has been working in Chaitanya Bharathi Institute of Technology, Hyderabad, in various capacities, since 1986. Presently, he is the Professor \& Head of Information Technology the Department. His research interests include Image Processing \& Computer Vision, Patter Recognition, Data Mining, AI, ANN, Bid Data Analytics, etc. He has guided more than fifty ME/MTech Projects. Several scholars from JNTUH and Osmania University have been working under him for $\mathrm{PhD}$ and one Scholar was awarded $\mathrm{PhD}$ degree. He has published more than 70 research publications in various national and international conferences and journals. He is a member of various Professional societies like ISTE, ISME, IEEE, IEEE-CIS, etc. He was honored with Best Teacher Award for his extraordinary contribution in teaching field during the year 2017-18 by Bharath Talents Association.

journals.

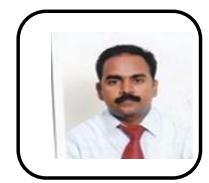

Dr D L Srinivas Reddy, is working as associate professor in IT dept in CBIT, Hyderabad. His research interests includes data analytics, machine learning and deep learning. He published several papers in reputed journals. 